\title{
Stabilization and Regression of Coronary Plaques Treated With Pitavastatin Proven by Angioscopy and Intravascular Ultrasound - The TOGETHAR Trial -
}

\author{
Kazuhisa Kodama, MD; Sei Komatsu, MD; Yasunori Ueda, MD; Tadateru Takayama, MD; \\ Jyunji Yajima, MD; Shinsuke Nanto, MD; Hiroshi Matsuoka, MD; \\ Satoshi Saito, MD; Atsushi Hirayama, MD
}

\begin{abstract}
Background: Few studies have serially monitored the change of coronary plaque after statin therapy using multiple plaque imaging modalities.
\end{abstract}

\begin{abstract}
Methods and Results: A prospective open-label trial was performed to assess coronary plaque regression and stabilization following 52 weeks of pitavastatin treatment $(2 \mathrm{mg} / \mathrm{day})$. Coronary segments that included the most diseased plaque of 90 patients determined on angioscopy were analyzed using intravascular ultrasound (IVUS). The yellow grade of each plaque of 46 patients who had matched angioscopy and IVUS data was evaluated on angioscopy. Low-density lipoprotein-cholesterol (LDL-C) was reduced $34.5 \%(145.0 \pm 24.0 \mathrm{mg} / \mathrm{dl}$ to $93.6 \pm$ $22.6 \mathrm{mg} / \mathrm{dl}, \mathrm{P}<0.001)$, and high-density lipoprotein cholesterol increased $17.8 \%(44.9 \pm 11.1 \mathrm{mg} / \mathrm{dl}$ to $51.9 \pm 11.7 \mathrm{mg} / \mathrm{dl}$, $\mathrm{P}<0.001)$. Yellow grade decreased $(2.9 \pm 0.8$ to $2.6 \pm 0.7, \mathrm{P}=0.040)$ during 52 weeks. The reduction of yellow grade was not correlated with the LDL-C level at 52 weeks or its change. The change of yellow grade was inversely correlated with maximum yellow grade at baseline. Percent atheroma volume on IVUS did not change during 52 weeks, but its change for 52 weeks was significantly correlated with LDL-C level at 52 weeks (Spearman's rank correlation coefficient $0.312, \mathrm{P}=0.035$ ).
\end{abstract}

Conclusions: Fixed dose pitavastatin stabilized vulnerable coronary plaques by the reduction of yellow grade without significant reduction of plaque volume. The stabilization and regression of atherosclerotic plaques by statin may differ, but both nonetheless contribute to the reduction of cardiovascular events (UMIN Clinical Trials Registry UMIN000001107). (Circ J 2010; 74: 1922-1928)

Key Words: Coronary angioscopy; Intravascular ultrasound; Pitavastatin; Plaque regression; Plaque stabilization

$\mathbf{L}$ owering of low-density lipoprotein-cholesterol (LDL-C) with 3-hydroxy-3-methylglutaryl-coenzyme A (HMG-CoA) reductase inhibitors (statins) has been shown to reduce cardiovascular mortality and morbidity. ${ }^{1-4}$ Many multicenter trials found that statin therapy produced plaque regression and stabilization as seen on intravascular ultrasound (IVUS)..$^{5-8}$

Coronary angioscopy gives a full-color, 3-D perspective of the intracoronary surface morphology, and reasonably accurate information regarding a specific lesion. ${ }^{9-20}$ Coronary angioscopy can detect some features of vulnerable plaque effectively. ${ }^{12}$ Among these, the yellow color intensity of plaques on coronary angioscopy, determined by the thickness of the fibrous cap, is known to be associated with plaque vulnerability. Particularly, a high grade of yellow plaque may indicate a large lipid core and thin fibrous cap, suggesting a high risk of rupture. ${ }^{12}$

This study group previously had prospectively investigated the qualitative and quantitative changes in coronary plaques in hypercholesterolemic patients with coronary artery disease receiving atorvastatin titrated to achieve LDL-C levels of $\leq 100 \mathrm{mg} / \mathrm{dl}$ (TWINS study). ${ }^{13}$ In that study, angioscopic

Received January 20, 2010; revised manuscript received April 20, 2010; accepted April 21, 2010; released online July 8, 2010 Time for primary review: 22 days

Department of Cardiology, Amagasaki Central Hospital, Amagasaki (K.K., S.K.); Division of Cardiology, Department of Medicine, Nihon University School of Medicine, Tokyo (K.K., T.T., S.S., A.H.); Department of Cardiology, Cardiovascular Institute Hospital, Tokyo (J.Y.); Cardiovascular Division, Osaka Police Hospital, Osaka (Y.U.); Advanced Cardiovascular Therapeutics, Osaka University Graduate School of Medicine, Suita (S.N.); and Department of Cardiology, Ehime Prefectural Imabari Hospital, Imabari (H.M.), Japan

Mailing address: Sei Komatsu, MD, PhD, FACC, Department of Cardiology, Amagasaki Central Hospital, 1-12-1 Shio-e, Amagasaki 661-0976, Japan. E-mail: plaquemap@yahoo.co.jp

ISSN-1346-9843 doi:10.1253/circj.CJ-10-0038

All rights are reserved to the Japanese Circulation Society. For permissions, please e-mail: cj@j-circ.or.jp 
loss of yellow color occurred prior to volumetric plaque regression on atorvastatin therapy. The different time courses of these changes suggested that the improvement in plaque characteristics occurred early, whereas atheroma volume reduction occurred over a prolonged period of time.

The aim of the present study was therefore to investigate the effects of fixed dose $(2 \mathrm{mg})$ pitavastatin on the stabilization and regression of yellow plaque on coronary angioscopy and IVUS (TOGETHAR) during a period of 52 weeks.

\section{Methods}

\section{Study Design and Patient Population}

The study was designed as a prospective open-label trial to assess the effects of 52 weeks of treatment with pitavastatin on angioscopy and IVUS of quantitative or qualitative changes in atherosclerosis at yellow plaques.

Patients with suspected or known coronary artery disease (CAD) complicated by hypercholesterolemia, with a fasting LDL-C level $\geq 120 \mathrm{mg} / \mathrm{dl}$ within 4 weeks of study initiation were initially screened. After angioscopy, patients having yellow plaque, color intensity higher than grade 2 were enrolled.

Exclusion criteria were administration of pitavastatin before enrollment, intolerance or contraindication to statin, acute myocardial infarction, homozygous familial hypercholesterolemia undergoing apheresis, presence of malignancies or mental disorder within 5 years prior to enrollment, progressive hepatic disorder, treatment with dialysis, serum creatinine $\geq 2 \mathrm{mg} / \mathrm{dl}$ or creatine kinase $\geq 500 \mathrm{IU} / \mathrm{L}$, and uncontrolled diabetes with hemoglobin $A_{1 c} \geq 8.0 \%$.

All eligible patients received a daily dose of oral pitavastatin $2 \mathrm{mg}$ /day for 52 weeks. Coronary angiography was performed at week 0 (baseline) and at week 52 .

The study protocol was approved by the Institutional Review Boards of Osaka Police Hospital. All patients provided written informed consent before participation.

\section{Angioscopy}

Immediately after coronary angiography, baseline angioscopy images were obtained with a Fiber Imaging System FT-201 (FiberTech Co Ltd, Tokyo, Japan) and the Console (Intertec Medicals Co Ltd, Osaka, Japan), using a previously described technique. ${ }^{9-20}$ From these images, lesions located in major coronary arteries were selected for the main angioscopy examination. Lesions close to culprit plaques treated by percutaneous coronary intervention, that is, those within $5 \mathrm{~mm}$, were excluded. Percent stenosis was not used for selection of the lesion for angioscopy because plaque color is not necessarily related to it. Images were recorded on a digital videotape and yellow plaque graded using a 5-point scale: 0 , not yellow at all; 1, pale yellow; 2, yellow; 3, deep yellow; 4, bright yellow. ${ }^{13}$ Already ruptured plaque was excluded. One maximum yellow grade per patient at baseline was determined. Vulnerable plaque was defined as $\geq$ grade 2 .

The positions of the plaques were identified on fluoroscopy by determining the location of the fiberscope tip and making a plaque map. The angioscopically most progressed plaque of each patient was identified. At week 52 the position of the maximum yellow grade at baseline was observed on angioscopy, in reference to the positions of side branches used as landmarks at baseline. For determining the color grade, all angioscopy images were reviewed by 2 independent investigators who were unaware of the patients' data and the time of image acquisition. The discordant plaques were intensively reevaluated by the reviewers, but if there was

\begin{tabular}{|lc|}
\hline \multicolumn{1}{|c|}{ Table 1. Baseline Patient Characteristics } \\
$\mathrm{n}$ & $\mathbf{n}(\%)$ \\
Age (years), mean $\pm \mathrm{SD}$ & 46 \\
Gender $(\mathrm{F} / \mathrm{M})$ & $62.5 \pm 10.3$ \\
Body mass index $\left(\mathrm{kg} / \mathrm{m}^{2}\right)$, mean $\pm \mathrm{SD}$ & $7 / 39$ \\
History of hypertension & $24.7 \pm 3.0$ \\
History of diabetes mellitus & $37(80.4)$ \\
History of angina pectoris & $16(34.8)$ \\
History of myocardial infarction & $18(39.1)$ \\
Concomitant medications & $24(52.2)$ \\
Aspirin & \\
Angiotensin-converting enzyme inhibitors & $46(100)$ \\
Angiotensin receptor blockers & $7(15.2)$ \\
$\beta$-blockers & $31(67.4)$ \\
Calcium blockers & $22(47.8)$ \\
History of statin use & $20(43.5)$ \\
\hline
\end{tabular}

still discordance between them after the second evaluation, they further evaluated the images individually until they reached consensus. During this process, the images were completely blinded to the investigators in terms of patient identity and time of acquisition.

\section{IVUS Image Acquisition}

IVUS measurements were performed with a $2.6-\mathrm{F} 40-\mathrm{MHz}$ Atlantis Pro IVUS catheter and Clear View Imaging System (Boston Scientific Corporation, Natick, MA, USA). The IVUS probe was advanced to a side branch located distal to the target lesion and images were obtained during automatic pullback at a rate of $0.5 \mathrm{~mm} / \mathrm{s}$ and recorded on s-VHS videotape.

Based on the plaque map in which yellow plaque was located on angioscopy, IVUS volumetry of the target lesions were conducted using at least 10-mm segment (IVUS analysis segment) at lesion sites as the unit of analysis at week 0 and week 52. At baseline examination the IVUS analysis segment was determined to include the plaque with the maximum yellow intensity, based on the plaque map and the position of a side branch. At the examinations performed at week 52, the IVUS analysis segment was set using a side branch as a landmark to ensure that the same position was imaged on IVUS. The boundary of the lumen and external elastic membrane was traced semi-automatically on digitized cross-sections of the IVUS analysis segment every $0.1 \mathrm{~mm}$ using a 3-D analysis system (echoPlaque2, INDEC Systems, CA, USA). Percent atheroma volume (PAV) was calculated as described in previous reports. ${ }^{3,11}$

IVUS volumetry was performed at a core laboratory in the Nihon University School of Medicine by an operator who was not involved in the medical care of the patients. Two investigators independent of the core laboratory and blinded to the patients' identity and time of image acquisition individually evaluated the images, including the accuracy of target lesion analysis at baseline and at week 52 .

\section{Data Matching in the Primary Analysis}

The coronary segments $\geq 10 \mathrm{~mm}$ that included the angioscopically most progressed plaque of each patient were analyzed on IVUS. Data for a plaque that had undergone angioscopic colorimetry and IVUS volumetry at baseline and at week 52 were treated as "matched data". Maximum yellow grade per 


\begin{tabular}{|c|c|c|c|c|}
\hline & Baseline & Week 52 & $\%$ change & $P$ value \\
\hline LDL-C (mg/dl) & $145.0 \pm 24.0$ & $93.6 \pm 22.6$ & $-34.5 \pm 16.2$ & $<0.001$ \\
\hline $\mathrm{HDL}-\mathrm{C}(\mathrm{mg} / \mathrm{dl})$ & $44.9 \pm 11.1$ & $51.9 \pm 11.7$ & $17.8 \pm 19.9$ & $<0.001$ \\
\hline LDL-C/HDL-C & $3.4 \pm 1.0$ & $1.9 \pm 0.7$ & $-43.1 \pm 16.8$ & $<0.001$ \\
\hline Apolipoprotein A-1 (mg/dl) & $118.5 \pm 21.6$ & $134.8 \pm 22.2$ & $15.5 \pm 18.4$ & $<0.001$ \\
\hline Apolipoprotein B (mg/dl) & $118.4 \pm 20.2$ & $83.4 \pm 18.9$ & $-28.9 \pm 14.5$ & $<0.001$ \\
\hline $\mathrm{TC}(\mathrm{mg} / \mathrm{dl})$ & $224.8 \pm 31.7$ & $172.7 \pm 25.3$ & $-22.3 \pm 12.3$ & $<0.001$ \\
\hline $\mathrm{TG}(\mathrm{mg} / \mathrm{dl})$ & $188.1 \pm 108.4$ & $148.7 \pm 84.8$ & $-12.8 \pm 40.3$ & 0.003 \\
\hline RLP-C (mg/dl) & $8.3 \pm 6.0$ & $5.0 \pm 3.2$ & $-30.4 \pm 32.7$ & $<0.001$ \\
\hline
\end{tabular}

LDL-C, low-density lipoprotein-cholesterol; HDL-C, high-density lipoprotein-cholesterol; TC, total cholesterol; TG, triglycerides; RLP-C, remnant-like particle-cholesterol.

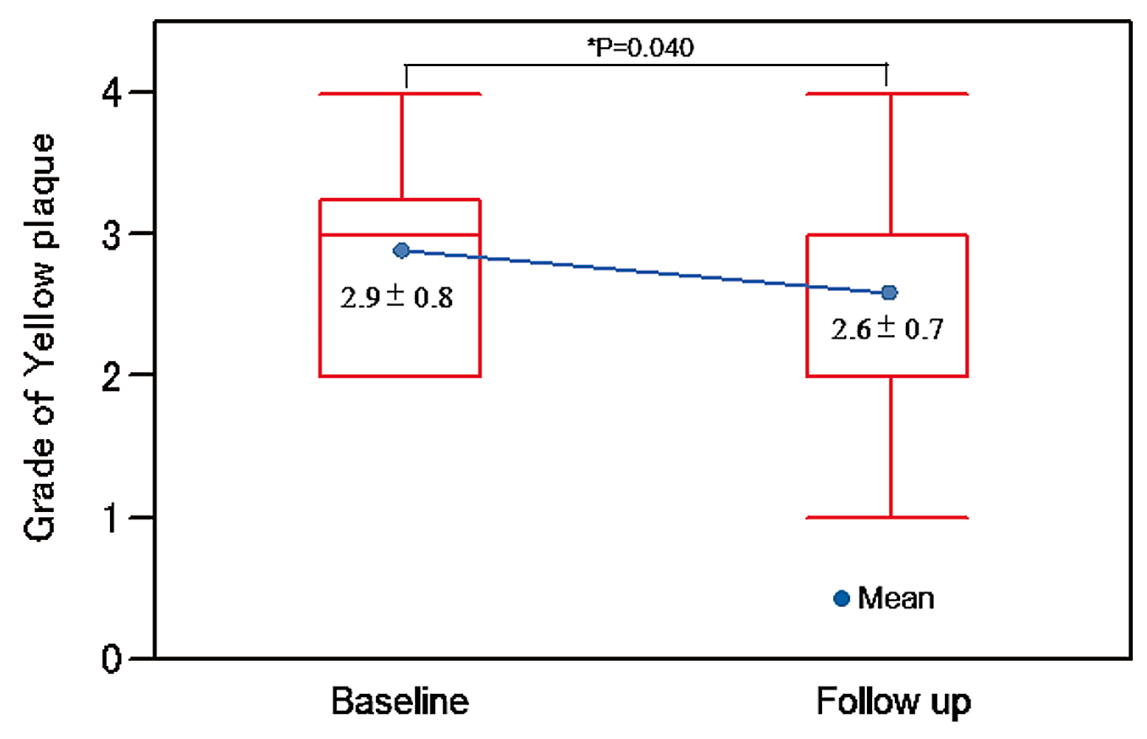

Figure 1. Comparison of yellow grade at baseline and week 52 . The yellow grade was significantly reduced from $2.9 \pm 0.8$ (95\% confidence interval $(\mathrm{Cl})=2.7-3.1)$ at baseline to $2.6 \pm 0.7(95 \% \mathrm{Cl}=2.4$ $2.8, \mathrm{P}<0.04)$ at week 52. *Wilcoxon signed rank test.

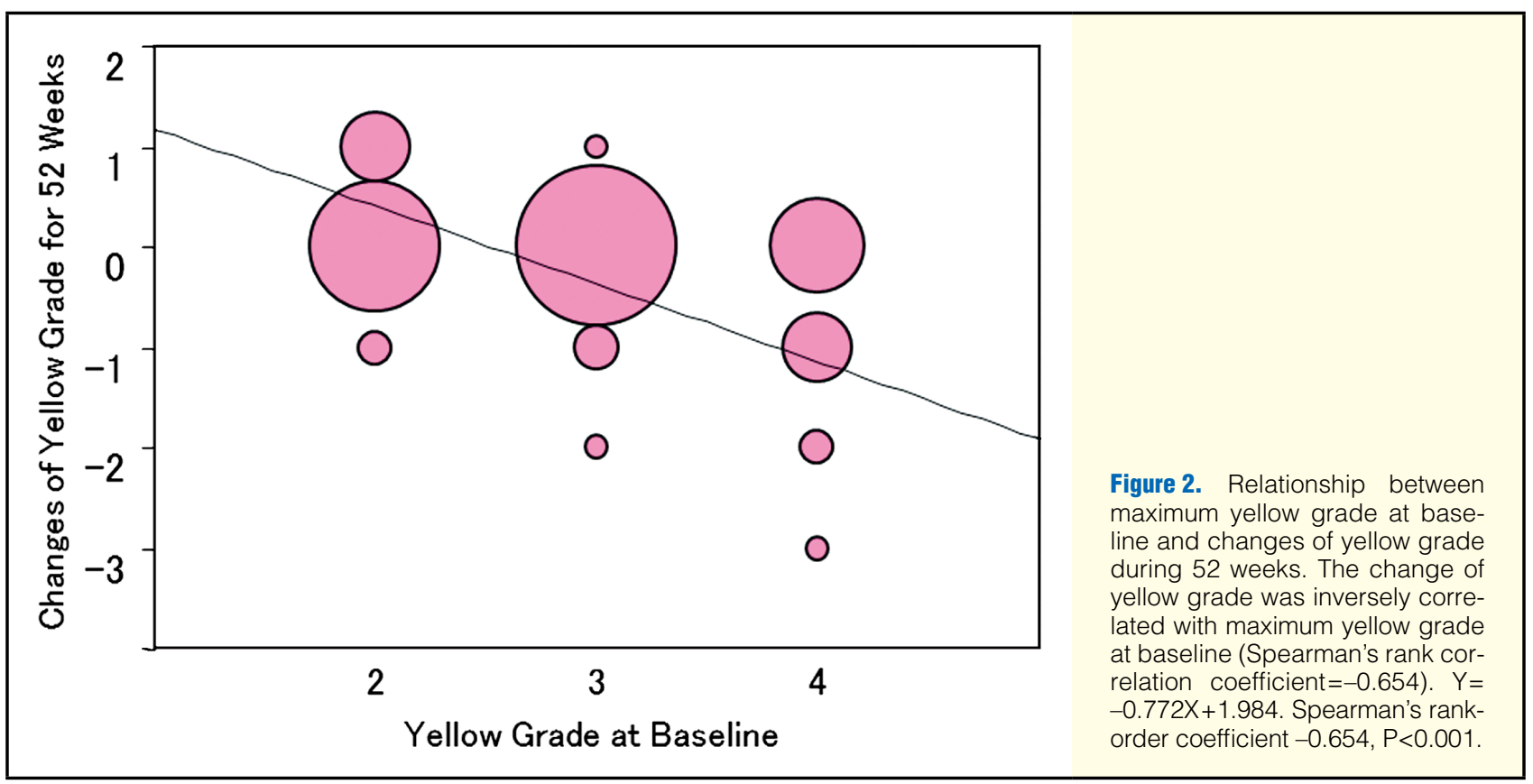




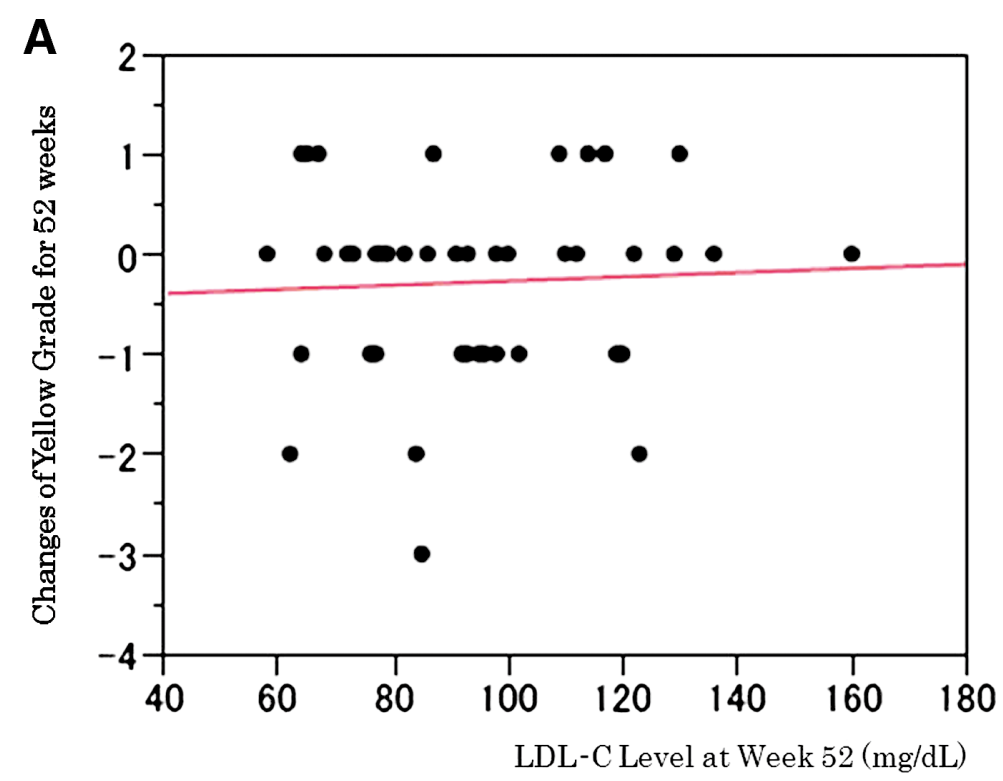

B

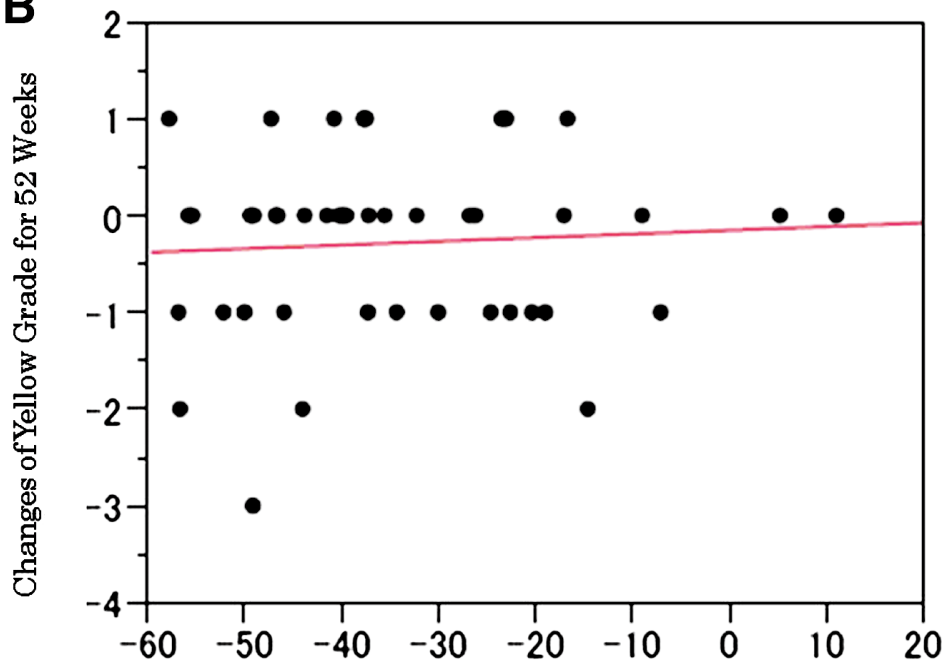

Changes of Serum LDL-C Level for 52 Weeks (\%)

C

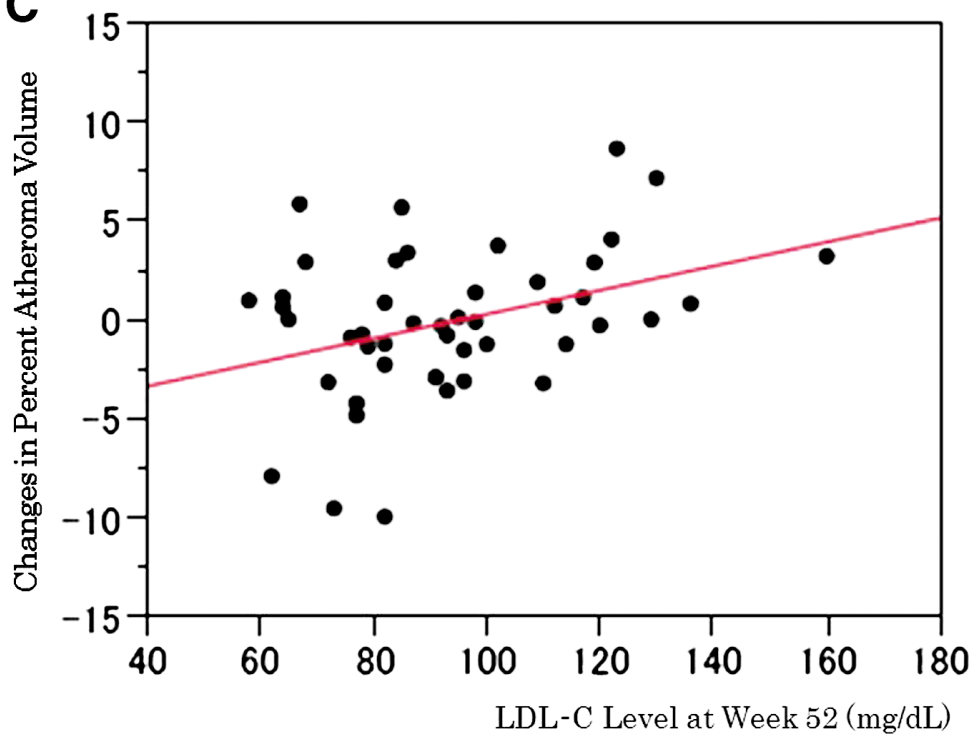

Figure 3. (A) There was no correlation between serum low-density lipoprotein-cholesterol (LDL-C) level at week 52 and change of yellow grade after 52 weeks. $Y=0.0021 X-$ 0.481 . Spearman's rank-order coefficient $-0.003, P=0.983$. (B) There was no correlation between change of serum LDL-C level after 52 weeks and change of yellow grade after 52 weeks. $Y=0.0038 X-0.152$. Spearman's rank-order coefficient $0.036, P=0.814$. (C) Change of percent atheroma volume was correlated with LDL-C level at week 52 (Pearson correlation coefficient $=0.364$, Spearman's rank correlation coefficient $=0.312$ ). $Y=0.061 X-$ 5.820. Spearman's rank-order coefficient 0.312, $\mathrm{P}=0.035$. 


\begin{tabular}{lcccccc}
\hline \multicolumn{7}{l}{ Table 3. Percent Atheroma Volume vs Time } \\
Median & Mean & SD & Minimum & Maximum & Wilcoxon test \\
Baseline & 47.0 & 47.2 & 8.6 & 23.5 & 63.1 & \\
Week 52 & 46.3 & 47.1 & 8.9 & 22.2 & 61.4 & \\
Change & -0.1 & -0.1 & 3.8 & -10.0 & 8.6 & $\mathrm{P}=0.859$ \\
\hline
\end{tabular}

patient at week 52 was compared with baseline. When multiple yellow plaques were included in a single 20 -mm IVUS analysis segment, the maximum value of the color grades was used. For this reason, if any yellow plaques observed at baseline in an IVUS analysis segment were unable to be reevaluated angioscopically at week 52 because of procedural difficulties, the set of plaques and the IVUS analysis segment in question were excluded from the analysis.

\section{Statistical Analysis}

Because this study was designed as preliminary exploratory research to investigate the relationship between quantitative and qualitative changes of coronary atherosclerotic plaques, the number of patients to be screened was planned as 100 . The Wilcoxon signed-rank test was used for comparison of plaque color grade between the data before and after treatment. With respect to the IVUS variables, the paired t-test was used to compare the data. The t-test was performed to compare LDL-C and high-density lipoprotein-cholesterol (HDLC) levels before and after treatment. In all tests, $\mathrm{P}<0.05$ was considered statistically significant.

\section{Results}

\section{Patient Population}

Between November 2004 and June 2006, 100 patients who gave informed consent to participate in the study and were satisfied with the entry criteria were screened. A total of 90 patients were followed for 52 weeks and, of these 90 patients, 78 patients had yellow plaque $\geq$ grade 2 , suggesting vulnerable plaque. Among them, 46 patients had matched angioscopy and IVUS data. Therefore, 46 segments that included plaques of maximum yellow grade on angioscopy were used in the present study. The baseline patient characteristics are shown in Table 1.

\section{Change in Laboratory Data (Table 2)}

LDL-C was reduced $34.5 \%(145.0 \pm 24.0 \mathrm{mg} / \mathrm{dl}$ to $93.6 \pm$

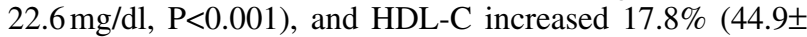
$11.1 \mathrm{mg} / \mathrm{dl}$ to $51.9 \pm 11.7 \mathrm{mg} / \mathrm{dl}, \mathrm{P}<0.001)$. The mean LDL-C/ HDL-C ratio was reduced from 3.4 at baseline to 1.9 at week 52. High sensitive C-reactive protein was reduced from $2.2 \pm$ $4.6 \mathrm{mg} / \mathrm{L}$ at baseline to $0.9 \pm 1.2 \mathrm{mg} / \mathrm{L}$ at week $52(\mathrm{P}<0.011)$.

\section{Changes in Angioscopy Findings}

The yellow grade was significantly reduced from $2.9 \pm 0.8$ (95\% confidence interval $(\mathrm{CI})=2.7-3.1)$ at baseline to $2.6 \pm$ $0.7(95 \% \mathrm{CI}=2.4-2.8, \mathrm{P}<0.04)$ at week 52 (Figure 1). The change of yellow grade was inversely correlated with maximum yellow grade at baseline (Spearman's rank correlation coefficient $-0.654, \mathrm{P}<0.001$; Figure 2 ). There was no correlation between serum LDL-C level at week 52 and changes of yellow grade during 52 weeks (Figure 3A). There was also no correlation between changes of serum LDL-C level after 52 weeks and changes of yellow grade after 52 weeks (Figure 3B).

\section{Changes in IVUS Findings}

The length of the measured segment was $17.55 \pm 8.60 \mathrm{~mm}$, ranging from $10.00 \mathrm{~mm}$ to $52.83 \mathrm{~mm}$. PAV at baseline $(47.2 \pm$ $8.6 \%)$ did not decrease significantly $(-0.1 \pm 3.8 \% ; 95 \% \mathrm{CI}=$ -1.2 to 1.0$)$ by week $52(47.1 \pm 8.9 \%, \mathrm{P}=0.859$; Table 3$)$. The change of PAV was correlated with LDL-C at week 52 (Spearman's rank correlation coefficient 0.312, $\mathrm{P}=0.035$; Figure 3C).

\section{Adverse Events}

Major adverse clinical events consisted of 1 occurrence of unstable angina and 1 occurrence of acute heart failure. Other adverse drug effects were 2 occurrences of hypertension, 2 of gamma-glutamyltransferase increase, 1 of eczema, 1 of blood creatine phosphokinase increase, 1 occurrence of myoglobin blood increase, 1 of glucose urine, 1 of arthralgia, 1 of diabetes mellitus, 1 occurrence of alanine aminotransferase increase, and 1 occurrence of aspartate aminotransferase increase.

\section{Discussion}

Pathologically, plaque rupture and acute thrombotic occlusion occur at the site of vulnerable plaques, which contain lipid-rich plaque covered by a thin fibrous cap. ${ }^{21,22}$ A previous study comparing angioscopy and optical coherence tomography in vivo demonstrated that more intensely yellow plaque has a thinner fibrous cap. ${ }^{23}$ This close relation between yellow color intensity and the thickness of the fibrous cap has also been reported in an ex vivo study, using integrated backscatter IVUS and angioscopy on tissue isolated autopsied specimen. ${ }^{24}$ Pan-coronary screening using angioscopy showed that coronary arteries have multiple yellow plaques in patients with acute coronary syndrome, ${ }^{19}$ and the number of yellow plaques correlated with the risk of acute coronary syndrome. ${ }^{20}$ The current prospective trial using angioscopy for coronary vulnerable plaques during 52 weeks of pitavastatin therapy showed reduction of plaque yellow grade. The mean change in angioscopic yellow grade was not large, a 0.3 -point decrease after 52 weeks; but plaques of higher yellow grade at baseline tend to be stabilized more. This implies that highly vulnerable plaques were stabilized effectively by pitavastatin and may result in the prevention of cardiac event and improvement of prognosis in patients with coronary heart disease.

Plaque regression is thought to involve a different mechanism from the reduction of cardiovascular events by treatment with statins. Based on the previous reports, plaque regression may be dependent on both the type of coronary artery disease and the decrease in LDL-C. Plaque regression occurred more commonly in patients with acute coronary syndrome ${ }^{25,26}$ rather than in patients with stable angina. ${ }^{5}$ None of the present study patients had acute coronary syndrome, and the present results on IVUS did not show evidence of plaque reduction. Because a fixed dose of pitavastatin was used according to the present study protocol, the decrease in LDL-C level was small. The achieved LDL- $\mathrm{C}$ level after treatment in the 


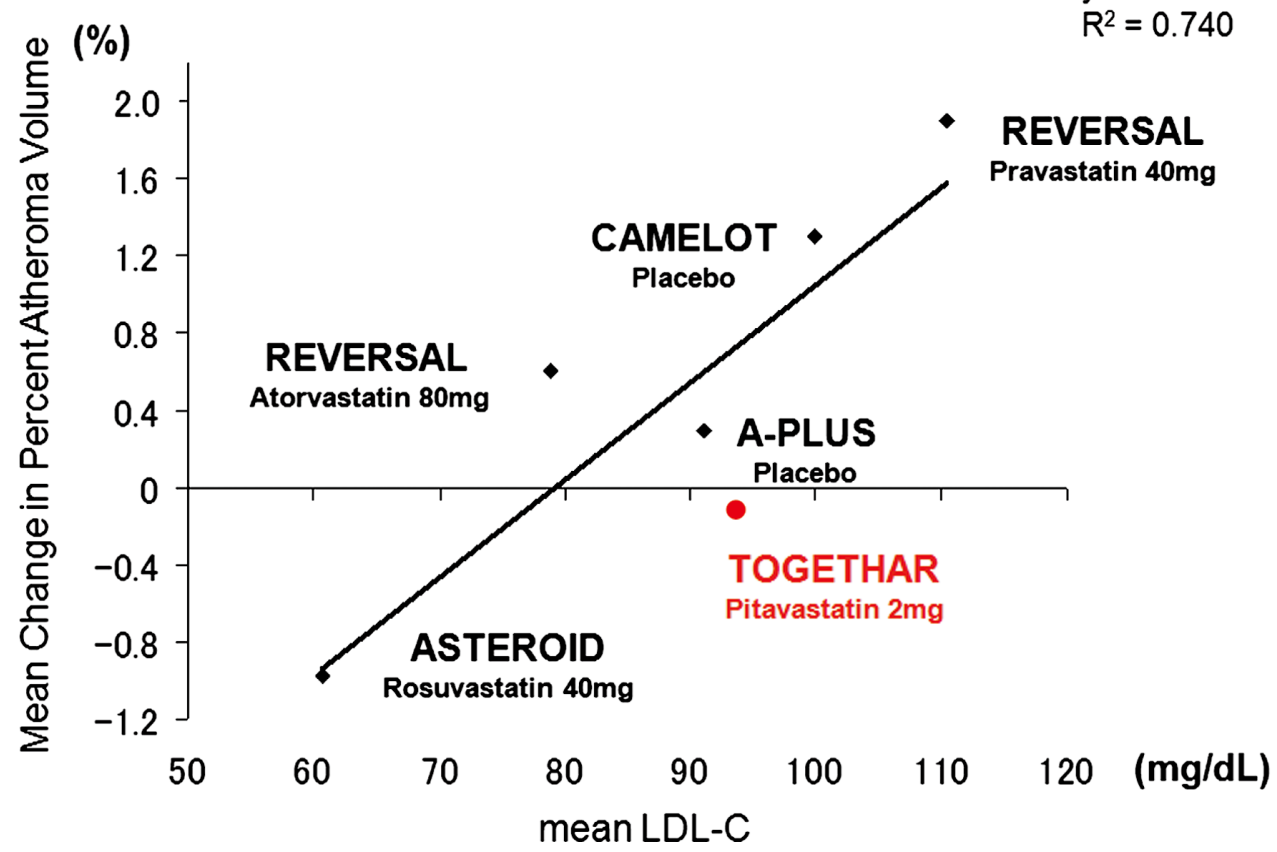

Figure 4. Relationship between mean low-density lipoprotein-cholesterol (LDL-C) levels and mean change in percent atheroma volume for intravascular ultrasound trials. ${ }^{6}$

present study was $90 \mathrm{mg} / \mathrm{dl}$, which was higher than that in the TWINS study. This might explain the insignificant plaque reduction. In the previous TWINS study, no patient was treated with statin prior to initiation of atorvastatin administration, but $8.7 \%$ of patients were already treated with another statin other than pitavastatin before enrollment in that study. Previous statin treatment might have made plaques resistant to regression by statin treatment in that study. In the COSMOS study, plaque regression was minimal in the previous statin treatment group compared to the de novo statin treatment group. ${ }^{27}$ Significant plaque progression was not observed in the present study and the result was comparable to the previous large-scale trial and compatible with the curve shown in Figure 4.

Some previous reports described that plaque stabilization and regression did not occur simultaneously. ${ }^{24,28}$ Hirayama et al reported that plaque stabilization occurred prior to plaque regression in the TWINS study. ${ }^{13}$ These results suggested that plaque stabilization and plaque regression reflect independent processes mediated by different mechanisms. In the present study the reduction of PAV determined on IVUS had a significant correlation with the reduction of LDL-C level. This correlation was also reported in the previous large-scale trials, suggesting that the reduction of LDL-C change might alter lipid transport from plaque to blood. In contrast, the reduction of plaque color intensity following treatment was not correlated with the LDL-C level at week 52 or change of LDL-C level. The change of plaque color might be an independent mechanism from the reduction of LDL-C. The early change of plaque color might explain the early benefit as shown in MIRACL ${ }^{2}$ or PROVE-IT. ${ }^{29}$ In PROVE-IT, the early benefit of atorvastatin compared to pravastatin was explained by the difference of anti-inflammatory effects between 2 statins. Using an animal model, Yokoyama et al demonstrated the anti-inflammatory effect of pitavastatin, inhibiting neointimal hyperplasia after stenting by a reduction of inflammatory reactions. ${ }^{30}$ Recently, Takarada et al reported that lipid-lowering therapy with statin for 9 months after the onset of acute myocardial infarction significantly increased the fibrous cap thickness in patients with hyperlipidemia. ${ }^{31}$ Taking these results into consideration, the change of plaque color might be due to anti-inflammatory effects of pitavastatin. There was no evidence for this in the present study, and further studies are needed to clarify the mechanism contributing to the plaque stabilization shown by the color changes.

A higher dose of pitavastatin with greater reduction of LDL-C level might be required for significant plaque regression as shown in the JAPAN-ACS study, ${ }^{26}$ but some reports implied that early coronary events decreased without plaque regression by statin. ${ }^{2,29}$ Coronary angioscopy may play a role in this explanation. Plaque stabilization on angioscopy and subsequent plaque regression on IVUS occurred after atorvastatin therapy in the TWINS study. ${ }^{13}$ The present results demonstrated that plaque stabilization on angioscopy occurred without plaque regression following treatment with $2 \mathrm{mg}$ pitavastatin. Moreover, stabilization was independent from the LDL-C level at week 52 and the change of LDL-C level after 52 weeks. These results including those from previous studies suggest that pitavastatin may contribute to prevent cardiovascular events by multiple mechanisms leading to plaque regression dose dependently, and also to early yellow grade reduction dose independently.

\section{Conclusions}

Despite the higher age of the present patients, pitavastatin 
therapy stabilized coronary vulnerable plaque by diminishing yellow color intensity observed on angioscopy. Stabilization was independent of the LDL-C level at week 52 and the difference of PAV that might be related to the change of LDL-C level. These results suggest that concomitant monitoring on angioscopy and IVUS for plaque stabilization and regression is important and that the mechanisms underlying the stabilization and regression of atherosclerotic plaques by statins may differ but contribute to the reduction of cardiovascular events.

\section{Acknowledgments}

This study was funded by Kowa Pharmaceuticals.

\section{Disclosure}

There are no conflicts of interest.

\section{References}

1. Cannon CP, Braunwald E, McCabe CH, Rader DJ, Rouleau JL, Belder R, et al; Pravastatin or Atorvastatin Evaluation and Infection Therapy-Thrombolysis in Myocardial Infarction 22 Investigators. Intensive versus moderate lipid lowering with statins after acute coronary syndromes. N Engl J Med 2004; 350: 1495 - 1504.

2. Schwartz GG, Olsson AG, Ezekowitz MD, Ganz P, Oliver MF, Waters D, et al; Myocardial Ischemia Reduction with Aggressive Cholesterol Lowering (MIRACL) Study Investigators. Effects of atorvastatin on early recurrent ischemic events in acute coronary syndromes: The MIRACL study: A randomized controlled trial. JAMA 2001; 285: $1711-1718$.

3. Athyros VG, Kakafika AI, Papageorgiou AA, Araskevas KI, Tziomalos K, Anagnostis P, et al. Effects of statin treatment in men and women with stable coronary heart disease: A subgroup analysis of the GREACE Study. Curr Med Res Opin 2008; 24: $1593-1599$.

4. LaRosa JC, Grundy SM, Waters DD, Shear C, Barter P, Fruchart $\mathrm{JC}$, et al. Intensive lipid lowering with atorvastatin in patients with stable coronary disease. N Engl J Med 2005; 352: 1425-1435.

5. Nissen SE, Tuzcu EM, Schoenhagen P, Brown BG, Ganz P, Vogel RA, et al; REVERSAL Investigators. Effect of intensive compared with moderate lipid-lowering therapy on progression of coronary atherosclerosis: A randomized controlled trial. JAMA 2004; 291: $1071-1080$.

6. Nissen SE, Nicholls SJ, Sipahi I, Libby P, Raichlen JS, Ballantyne $\mathrm{CM}$, et al; ASTEROID Investigators. Effect of very high-intensity statin therapy on regression of coronary atherosclerosis: The ASTEROID trial. JAMA 2006; 295: 1556-1565.

7. Miyauchi K, Kimura T, Morimoto T, Nakagawa Y, Yamagishi M, Ozaki Y, et al. Japan assessment of pitavastatin and atorvastatin in acute coronary syndrome (JAPAN-ACS): Rationale and design. Circ J 2006; 70: 1624-1628.

8. Toi T, Taguchi I, Yoneda S, Kageyama M, Kikuchi A, Tokura M, et al. Early effect of lipid-lowering therapy with pitavastatin on regression of coronary atherosclerotic plaque: Comparison with atorvastatin. Circ J 2009; 73: 1466-1472.

9. Kodama K, Asakura M, Ueda Y, Yamaguchi O, Hirayama A. The role of plaque rupture in the development of acute coronary syndrome evaluated by the coronary angioscope. Intern Med 2000; 39: $333-335$.

10. Ueda Y, Asakura M, Hirayama A, Adachi T, Kodama K. Angioscopy of culprit lesions. Cardiologia 1997; 42: 827-832.

11. Ueda Y, Ohtani T, Shimizu M, Hirayama A, Kodama K. Assessment of plaque vulnerability by angioscopic classification of plaque color. Am Heart J 2004; 148: 333-335.

12. Naghavi M, Libby P, Falk E, Casscells SW, Litovsky S, Rumberger J, et al. From vulnerable plaque to vulnerable patient: A call for new definitions and risk assessment strategies: Part I. Circulation 2003; 108: 1664-1672.

13. Hirayama A, Saito S, Ueda Y, Takayama T, Honye J, Komatsu S, et al. Qualitative and quantitative changes in coronary plaque associated with atorvastatin therapy. Circ J 2009; 73: 718-725.

14. Komatsu S, Sato Y, Ueda Y, Achenbach S, Ebihara Y, Hirayama A, et al. Thrombotic occlusion proximal to plaque rupture in acute myocardial infarction; evaluation by intravascular ultrasound and coronary angioscopy. Int J Cardiol 2007; 123: e12-e14.

15. Komatsu S, Hirayama A, Ueda Y, Okuyama Y, Ogasawara N,
Kashiwase K, et al. Coronary ruptured plaque mimicking spontaneous coronary dissection in a young woman. Int J Cardiol 2006; 113: $288-289$.

16. Higo T, Ueda Y, Oyabu J, Okada K, Nishio M, Hirata A, et al. Atherosclerotic and thrombogenic neointima formed over sirolimus drug-eluting stent: An angioscopic study. JACC Cardiovasc Imaging 2009; 2: 616-624.

17. Oyabu J, Ueda Y, Ogasawara N, Okada K, Hirayama A, Kodama K. Angioscopic evaluation of neointima coverage: Sirolimus drug-eluting stent versus bare metal stent. Am Heart J 2006; 152: 1168 1174.

18. Ueda Y, Asakura M, Yamaguchi O, Hirayama A, Hori M, Kodama $\mathrm{K}$. The healing process of infarct-related plaques: Insights from 18 months of serial angioscopic follow-up. J Am Coll Cardiol 2001; 38: $1916-1922$.

19. Asakura M, Ueda Y, Yamaguchi O, Adachi T, Hirayama A, Hori M, et al. Extensive development of vulnerable plaques as a pan-coronary process in patients with myocardial infarction: An angioscopic study. J Am Coll Cardiol 2001; 37: 1284-1288.

20. Ohtani T, Ueda Y, Mizote I, Oyabu J, Okada K, Hirayama A, et al. Number of yellow plaques detected in a coronary artery is associated with future risk of acute coronary syndrome: Detection of vulnerable patients by angioscopy. J Am Coll Cardiol 2006; 47: 2194-2200.

21. Davies MJ, Thomas AC. Plaque fissuring: The cause of acute myocardial infarction, sudden ischemic death, and crescendo angina. Br Heart J 1985; 53: 363-373.

22. Brown BG, Zhao XQ, Sacco DE, Albers JJ. Lipid lowering and plaque regression: New insights into prevention of plaque disruption and clinical events in coronary disease. Circulation 1993; 87: $1781-1791$.

23. Kubo T, Imanishi T, Takarada S, Kuroi A, Ueno S, Yamano T, et al. Implication of plaque color classification for assessing plaque vulnerability: A coronary angioscopy and optical coherence tomography investigation. JACC Cardiovasc Interv 2008; 1: 74-80.

24. Kawasaki M, Sano K, Okubo M, Yokoyama H, Ito Y, Murata I, et al. Volumetric quantitative analysis of tissue characteristics of coronary plaques after statin therapy using three-dimensional integrated backscatter intravascular ultrasound. J Am Coll Cardiol 2005; 45: 1946-1953.

25. Okazaki S, Yokoyama T, Miyauchi K, Shimada K, Kurata T, Sato $\mathrm{H}$, et al. Early statin treatment in patients with acute coronary syndrome: Demonstration of the beneficial effect on atherosclerotic lesions by serial volumetric intravascular ultrasound analysis during half a year after coronary event: The ESTABLISH Study. Circulation 2004; 110: 1061 - 1068.

26. Hiro T, Kimura T, Morimoto T, Miyauchi K, Nakagawa Y, Yamagishi M, et al; JAPAN-ACS Investigators. Effect of intensive statin therapy on regression of coronary atherosclerosis in patients with acute coronary syndrome: A multicenter randomized trial evaluated by volumetric intravascular ultrasound using pitavastatin versus atorvastatin (JAPAN-ACS [Japan assessment of pitavastatin and atorvastatin in acute coronary syndrome] study). J Am Coll Cardiol 2009; 54: 293-302.

27. Takayama T, Hiro T, Yamagishi M, Daida H, Hirayama A, Saito S, et al; for the COSMOS Investigators. Effect of rosuvastatin on coronary atheroma in stable coronary artery disease: The multicenter Coronary Atherosclerosis Study Measuring Effects of Rosuvastatin Using Intravascular Ultrasound in Japanese Subjects (COSMOS). Circ J 2009; 73: 2110-2117.

28. Schartl M, Bocksch W, Koshyk DH, Voelker W, Karsch KR, Kreuzer J, et al. Use of intravascular ultrasound to compare effects of different strategies of lipid-lowering therapy on plaque volume and composition in patients with coronary artery disease. Circulation 2001; 104: 387-392.

29. Ray KK, Cannon CP, Cairns R, Morrow DA, Rifai N, Kirtane AJ, et al; PROVE IT-TIMI 22 Investigators. Relationship between uncontrolled risk factors and C-reactive protein levels in patients receiving standard or intensive statin therapy for acute coronary syndromes in the PROVE IT-TIMI 22 trial. J Am Coll Cardiol 2005; 46: $1417-1424$.

30. Yokoyama T, Miyauchi K, Kurata T, Satoh H, Daida H. Inhibitory efficacy of pitavastatin on the early inflammatory response and neointimal thickening in a porcine coronary after stenting. Atherosclerosis 2004; 174: $253-259$.

31. Takarada S, Imanishi T, Kubo T, Tanimoto T, Kitabata H, Nakamura N, et al. Effect of statin therapy on coronary fibrous-cap thickness in patients with acute coronary syndrome: Assessment by optical coherence tomography study. Atherosclerosis 2009; 202: $491-497$. 